\title{
The refinement and generalization of Hardy's inequality in Sobolev space
}

\author{
Xiaomin $\mathrm{Xue}^{1}$ and Fushan $\mathrm{Li}^{1,2^{*}}$
}

\section{"Correspondence:}

fushan99@163.com

${ }^{1}$ School of Mathematical Sciences, Qufu Normal University, Qufu, P.R.

China

${ }^{2}$ Center for Teaching Research and Evaluation, Qufu Normal University, Qufu, P.R. China

\section{Springer}

\begin{abstract}
In this paper, we refine the proof of Hardy's inequality in (Evans in Partial Differential Equations, 2010, Hardy in Inequalities, 1952) and extend Hardy's inequality from two aspects. That is to say, we extend the integral estimation function from $\frac{u}{|x|}$ to $\frac{u}{|x|^{\sigma}}$ with suitable $\sigma>0$ and extend the space dimension from $n \geq 3$ to $n \geq 2$. Hardy's inequality in (Evans in Partial Differential Equations, 2010, Hardy in Inequalities, 1952) is the special case of our results.
\end{abstract}

Keywords: Hardy's inequality; Integrate by part; Cauchy inequality; Divergence theorem

\section{Introduction}

It is well known that inequalities are important tools in classical analysis $[2-6,13,14$, $26-29,31-39,41-43,45]$. One application of inequalities is to study the properties of partial differential equations. Li and his coauthors [15-23] studied the global existence and uniqueness, limit behavior, uniform stability, and blow-up of solutions for partial differential equations by using various inequalities. Liu [11, 24, 25] showed the stability and convergence results of evolution equations and $\mathrm{Du}[8,9]$ studied obstacle problems by using various inequalities.

In recent decades, there have been many results on the extension and refinement of inequality [7, 10, 12, 30, 40,44]. Qin [30] summarized a large number of inequalities and applications, but Hardy's inequality was not included. The authors [7, 40] generalized the summation form Hardy inequality, Zhang [44] extended Hardy inequalities using Littlewood-Paley theory and nonlinear estimates method in Besov spaces, and the results improved and extended the well-known results in [1].

The first edition of classic textbook [10] does not contain Hardy's inequality, we see that the very significant Hardy's inequality

$$
\int_{B(o, r)} \frac{u^{2}}{|x|^{2}} d x \leq C \int_{B(o, r)}\left(|D u|^{2}+\frac{u^{2}}{r^{2}}\right) d x
$$

holds if $u \in H^{1}(B(o, r)), n \geq 3$, and $r>0$ in the second edition of [10]. The proof of Hardy's inequality given in $[10,12]$ is very ingenious, but it is not easy to master for the reader. Therefore, we refine the proof of Hardy's inequality for readers to grasp the essence of the proof and extend Hardy's inequality in Sobolev space from two aspects. That is to say, we

(c) The Author(s) 2018. This article is distributed under the terms of the Creative Commons Attribution 4.0 International License (http://creativecommons.org/licenses/by/4.0/), which permits unrestricted use, distribution, and reproduction in any medium, provided you give appropriate credit to the original author(s) and the source, provide a link to the Creative Commons license, and indicate if changes were made. 
extend the integral estimation function from $\frac{u}{|x|}$ to $\frac{u}{|x|^{\sigma}}$ with suitable $\sigma>0$ and extend the space dimension from $n \geq 3$ to $n \geq 2$. Hardy's inequality in $[10,12]$ is the special case of our results.

Let $B(o, r)$ be a closed ball in $\mathbf{R}^{n}$ with center $o$ and radius $r>0, x=\left(x_{1}, x_{2}, \ldots, x_{n}\right)$ be a vector in $B(o, r), v=\left(v_{1}, v_{2}, \ldots, v_{n}\right)=\left(\frac{x_{1}}{r}, \frac{x_{2}}{r}, \ldots, \frac{x_{n}}{r}\right)$ be the unit outward normal to $\partial B(o, r)$. $W^{k, p}(\Omega)$ and $H^{1}(\Omega)$ denote the Sobolev spaces. We write

$$
D u=\left(u_{x_{1}}, u_{x_{2}}, \ldots, u_{x_{n}}\right), \quad|x|=\left(x_{1}^{2}+x_{2}^{2}+\cdots+x_{n}^{2}\right)^{\frac{1}{2}} .
$$

In Sect. 2, we first recall Hardy's inequality, refine the proof for completeness, and state our main results. The proofs of the main results are given in Sect. 3.

\section{Main results}

Now, we present the global approximation theorem and Hardy's inequality in Sobolev space.

Lemma 2.1 ([10], Global approximation theorem) Assume that $\Omega$ is bounded and $\partial \Omega$ is $C^{1}$. Let $u \in W^{k, p}(\Omega)$ for some $1 \leq p<\infty$. Then there exist functions $u_{m} \in C^{\infty}(\bar{\Omega})$ such that

$$
u_{m} \rightarrow u \quad i n W^{k, p}(\Omega)
$$

Lemma 2.2 ([10,12], Hardy's inequality) Assume $n \geq 3$ and $r>0$. Let $u \in H^{1}(B(o, r))$. Then $\frac{u}{|x|} \in L^{2}(B(o, r))$ with the estimate

$$
\int_{B(o, r)} \frac{u^{2}}{|x|^{2}} d x \leq C \int_{B(o, r)}\left(|D u|^{2}+\frac{u^{2}}{r^{2}}\right) d x
$$

For readers to grasp the essence of the proof, we give the refined proof below.

Proof By the global approximation theorem Lemma 2.1, we may assume $u \in C^{\infty}(B(o, r))$. Noting that $D\left(\frac{1}{|x|^{\rho}}\right)=-\rho \frac{x}{|x|^{\rho+2}}$ for any $\rho>0$ and integrating by parts, we have

$$
\begin{aligned}
\int_{B(o, r)} \frac{u^{2}}{|x|^{2}} d x= & -\frac{1}{\rho} \int_{B(o, r)} u^{2} D\left(\frac{1}{|x|^{\rho}}\right) \cdot \frac{x}{|x|^{2-\rho}} d x \\
= & -\frac{1}{\rho} \int_{B(o, r)} u^{2} \sum_{i=1}^{n}\left(\frac{1}{|x|^{\rho}}\right)_{x_{i}} \frac{x_{i}}{|x|^{2-\rho}} d x \\
= & -\frac{1}{\rho} \int_{\partial B(o, r)} \sum_{i=1}^{n} u^{2} v_{i} \cdot \frac{x_{i}}{|x|^{2}} d S \\
& +\frac{1}{\rho} \int_{B(o, r)} \sum_{i=1}^{n} \frac{1}{|x|^{\rho}}\left(u^{2} \frac{x_{i}}{|x|^{2-\rho}}\right)_{x_{i}} d x \\
= & -\frac{1}{\rho r} \int_{\partial B(o, r)} u^{2} d S \\
& +\frac{1}{\rho} \int_{B(o, r)}\left[2 u D u \cdot \frac{x}{|x|^{2}}+(n+\rho-2) \frac{u^{2}}{|x|^{2}}\right] d x .
\end{aligned}
$$


Therefore

$$
(n-2) \int_{B(o, r)} \frac{u^{2}}{|x|^{2}} d x=-2 \int_{B(o, r)} u D u \cdot \frac{x}{|x|^{2}} d x+\frac{1}{r} \int_{\partial B(o, r)} u^{2} d S .
$$

For any $\varepsilon>0$, using the Cauchy inequality and Schwarz inequality, we obtain

$$
\begin{aligned}
-2 \int_{B(o, r)} u D u \cdot \frac{x}{|x|^{2}} d x & =-2 \int_{B(o, r)} \frac{u}{|x|} D u \cdot \frac{x}{|x|} d x \\
& \leq 2 \int_{B(o, r)} \frac{|u|}{|x|}|D u|\left|\frac{x}{|x|}\right| d x \\
& =2 \int_{B(o, r)} \frac{|u|}{|x|}|D u| d x \\
& \leq 2 \varepsilon \int_{B(o, r)} \frac{u^{2}}{|x|^{2}} d x+\frac{1}{2 \varepsilon} \int_{B(o, r)}|D u|^{2} d x .
\end{aligned}
$$

Fixing $\varepsilon>0$ such that $n-2-2 \varepsilon>0$, we conclude

$$
\int_{B(o, r)} \frac{u^{2}}{|x|^{2}} d x \leq C \int_{B(o, r)}|D u|^{2} d x+\frac{C}{r} \int_{\partial B(o, r)} u^{2} d S .
$$

According to the divergence theorem, we have

$$
\begin{aligned}
\int_{B(o, r)} \operatorname{div}\left(x u^{2}\right) d x & =\int_{\partial B(o, r)} x u^{2} \cdot v d S=\int_{\partial B(o, r)} u^{2} x \cdot \frac{x}{r} d S \\
& =r \int_{\partial B(o, r)} u^{2} d S .
\end{aligned}
$$

Using the Cauchy inequality and Schwarz inequality, we get

$$
\begin{aligned}
\int_{B(o, r)} \operatorname{div}\left(x u^{2}\right) d x & =\int_{B(o, r)}\left[u^{2} \operatorname{div}(x)+D\left(u^{2}\right) \cdot x\right] d x \\
& =\int_{B(o, r)}\left(n u^{2}+2 u D u \cdot x\right) d x \\
& \leq \int_{B(o, r)}\left(n u^{2}+u^{2}+|x|^{2}|D u|^{2}\right) d x \\
& \leq \int_{B(o, r)}\left[(n+1) u^{2}+r^{2}|D u|^{2}\right] d x .
\end{aligned}
$$

Combining (2.5) and (2.6), we obtain the trace inequality

$$
\frac{1}{r} \int_{\partial B(o, r)} u^{2} d S \leq C \int_{B(o, r)}\left(|D u|^{2}+\frac{u^{2}}{r^{2}}\right) d x
$$

Employing this inequality (2.7) in (2.4) finishes the proof of (2.1).

Under the circumstance, we extend the space dimension $n$ and parameter $\sigma$ in $\frac{u}{|x|^{\sigma}}$ of Hardy's inequality. Now we show our main results. 
Theorem 2.1 Assume $n \geq 2$ and $r>0, u \in H^{1}(B(o, r))$. Then, for $\sigma<\frac{n}{2}$, we have $\frac{u}{|x|^{\sigma}} \in$ $L^{2}(B(o, r))$ with the estimate as follows:

If $\sigma \leq 1$ and $\sigma<\frac{n}{2}$, we have

$$
\int_{B(o, r)} \frac{u^{2}}{|x|^{2 \sigma}} d x \leq C \int_{B(o, r)}\left(\frac{|D u|^{2}}{r^{2(\sigma-1)}}+\frac{u^{2}}{r^{2 \sigma}}\right) d x
$$

If $\sigma>1$ and $\sigma<\frac{n}{2}$, we have

$$
\int_{B(o, r)} \frac{u^{2}}{|x|^{2 \sigma}} d x \leq C \int_{B(o, r)}\left(\frac{|D u|^{2}}{|x|^{2(\sigma-1)}}+\frac{u^{2}}{r^{2 \sigma}}\right) d x
$$

Remark 2.1 Hardy's inequality (2.1) is the case of $\sigma=1$ and $n \geq 3$ in Theorem 2.1.

Remark 2.2 If $n=2$, then $\sigma<1$. $B(o, r)$ denotes a closed circular region with center $o$ and radius $r>0, \partial B(o, r)$ denotes a circle, and $\int_{B(o, r)} \cdots d S$ denotes curvilinear integration.

\section{Proofs of the main results}

In this section we show the proofs of the main results Theorem 2.1.

Proof For any $\rho>0$, since

$$
D\left(\frac{1}{|x|^{\rho}}\right)=-\rho \frac{x}{|x|^{\rho+2}}
$$

which implies

$$
\begin{aligned}
\frac{1}{|x|^{2 \sigma}} & =\left[-\rho \frac{x}{|x|^{\rho+2}}\right] \cdot\left[\left(-\frac{1}{\rho}\right) \frac{x}{|x|^{2 \sigma-\rho}}\right] \\
& =-\frac{1}{\rho} D\left(\frac{1}{|x|^{\rho}}\right) \cdot \frac{x}{|x|^{2 \sigma-\rho}} .
\end{aligned}
$$

By the global approximation theorem, we may assume $u \in C^{\infty}(B(o, r))$. Noting that (3.1) holds, we obtain

$$
\begin{aligned}
\int_{B(o, r)} \frac{u^{2}}{|x|^{2 \sigma}} d x= & -\frac{1}{\rho} \int_{B(o, r)} u^{2} D\left(\frac{1}{|x|^{\rho}}\right) \cdot \frac{x}{|x|^{2 \sigma-\rho}} d x \\
= & -\frac{1}{\rho} \int_{B(o, r)} \sum_{i=1}^{n}\left(\frac{1}{|x|^{\rho}}\right)_{x_{i}}\left(u^{2} \frac{x_{i}}{|x|^{2 \sigma-\rho}}\right) d x \\
= & -\frac{1}{\rho} \int_{\partial B(o, r)} \sum_{i=1}^{n} u^{2} v_{i} \cdot \frac{x_{i}}{|x|^{2 \sigma}} d S \\
& +\frac{1}{\rho} \int_{B(o, r)} \sum_{i=1}^{n} \frac{1}{|x|^{\rho}}\left(u^{2} \frac{x_{i}}{|x|^{2 \sigma-\rho}}\right)_{x_{i}} d x \\
= & -\frac{1}{\rho r^{2 \sigma-1}} \int_{\partial B(o, r)} u^{2} d S \\
& +\frac{1}{\rho} \int_{B(o, r)}\left[2 u D u \cdot \frac{x}{|x|^{2 \sigma}}+(n+\rho-2 \sigma) \frac{u^{2}}{|x|^{2 \sigma}}\right] d x
\end{aligned}
$$


Hence

$$
\begin{aligned}
(n-2 \sigma) \int_{B(o, r)} \frac{u^{2}}{|x|^{2 \sigma}} d x= & -2 \int_{B(o, r)} u D u \cdot \frac{x}{|x|^{2 \sigma}} d x \\
& +\frac{1}{r^{2 \sigma-1}} \int_{\partial B(o, r)} u^{2} d S .
\end{aligned}
$$

For any $\varepsilon>0$, using the Cauchy inequality and Schwarz inequality, we obtain

$$
\begin{aligned}
-2 \int_{B(o, r)} u D u \cdot \frac{x}{|x|^{2 \sigma}} d x & =-2 \int_{B(o, r)} \frac{u}{|x|^{\sigma}} D u \cdot \frac{x}{|x|^{\sigma}} d x \\
& \leq 2 \int_{B(o, r)} \frac{|u|}{|x|^{\sigma}}|D u|\left|\frac{x}{|x|^{\sigma}}\right| d x \\
& =2 \int_{B(o, r)} \frac{|u|}{|x|^{\sigma}}|D u| \frac{1}{|x|^{\sigma-1}} d x \\
& \leq 2 \varepsilon \int_{B(o, r)} \frac{u^{2}}{|x|^{2 \sigma}} d x \\
& +\frac{1}{2 \varepsilon} \int_{B(o, r)}|D u|^{2} \frac{1}{|x|^{2(\sigma-1)}} d x
\end{aligned}
$$

According to the divergence theorem, we have

$$
\int_{B(o, r)} \operatorname{div}\left(x u^{2}\right) d x=r \int_{\partial B(o, r)} u^{2} d S
$$

and using the Cauchy inequality and Schwarz inequality, we get

$$
\begin{aligned}
\int_{B(o, r)} \operatorname{div}\left(x u^{2}\right) d x & =\int_{B(o, r)}\left(n u^{2}+2 u D u \cdot x\right) d x \\
& \leq \int_{B(o, r)}\left(n u^{2}+u^{2}+|D u|^{2}|x|^{2}\right) d x \\
& \leq \int_{B(o, r)}\left[(n+1) u^{2}+r^{2}|D u|^{2}\right] d x
\end{aligned}
$$

which implies

$$
\frac{1}{r^{2 \sigma-1}} \int_{\partial B(o, r)} u^{2} d S \leq \frac{n+1}{r^{2 \sigma}} \int_{B(o, r)} u^{2} d x+\frac{1}{r^{2(\sigma-1)}} \int_{B(o, r)}|D u|^{2} d x .
$$

By substituting (3.4) and (3.5) into (3.3), fixing $\varepsilon$ such that $n-2 \sigma-2 \varepsilon>0$, we conclude

$$
\int_{B(o, r)} \frac{u^{2}}{|x|^{2 \sigma}} d x \leq C \int_{B(o, r)}\left[\frac{|D u|^{2}}{|x|^{2(\sigma-1)}}+\frac{|D u|^{2}}{r^{2(\sigma-1)}}+\frac{u^{2}}{r^{2 \sigma}}\right] d x .
$$

Therefore, from (3.6), for $n \geq 2$ and $\sigma<\frac{n}{2}$ :

if $\sigma \leq 1$, noting that

$$
\frac{|D u|^{2}}{|x|^{2(\sigma-1)}} \leq \frac{|D u|^{2}}{r^{2(\sigma-1)}}, \quad x \in B(o, r)
$$


we obtain

$$
\int_{B(o, r)} \frac{u^{2}}{|x|^{2 \sigma}} d x \leq C \int_{B(o, r)}\left(\frac{|D u|^{2}}{r^{2(\sigma-1)}}+\frac{u^{2}}{r^{2 \sigma}}\right) d x
$$

if $\sigma>1$, noting that

$$
\frac{|D u|^{2}}{|x|^{2(\sigma-1)}} \geq \frac{|D u|^{2}}{r^{2(\sigma-1)}}, \quad x \in B(o, r)
$$

we obtain

$$
\int_{B(o, r)} \frac{u^{2}}{|x|^{2 \sigma}} d x \leq C \int_{B(o, r)}\left(\frac{|D u|^{2}}{|x|^{2(\sigma-1)}}+\frac{u^{2}}{r^{2 \sigma}}\right) d x .
$$

The proof of Theorem 2.1 is completed.

\section{Conclusions}

In this paper, we refine the proof of Hardy's inequality for readers to grasp the essence of the proof and extend Hardy's inequality in Sobolev space from two aspects. That is to say, we extend the integral estimation function from $\frac{u}{|x|}$ to $\frac{u}{|x|^{\sigma}}$ with suitable $\sigma>0$ and extend the space dimension from $n \geq 3$ to $n \geq 2$. Hardy's inequality in $[10,12]$ is the special case of our results.

\section{Acknowledgements}

The authors would like to thank the referees for their valuable comments and suggestion.

\section{Funding}

This work was supported by the National Natural Science Foundation of China (No.11201258).

\section{Availability of data and materials}

Not applicable.

\section{Competing interests}

The authors declare that they have no competing interests.

\section{Authors' contributions}

The authors contributed equally to the writing of this paper. The authors read and approved the final manuscript.

\section{Publisher's Note}

Springer Nature remains neutral with regard to jurisdictional claims in published maps and institutional affiliations.

Received: 4 September 2018 Accepted: 22 November 2018 Published online: 28 November 2018

\section{References}

1. Cazenave, T.: Semilinear Schrödinger Equations. Courant Lecture Notes in Mathematics, vol. 10. Am. Math. Soc., Rhode Island (2003)

2. Chu, Y.M., Wang, G.D., Zhang, X.H.: Schur convexity and Hadamard's inequality. Math. Inequal. Appl. 13(4), 725-731 (2010)

3. Chu, Y.M., Wang, G.D., Zhang, X.H.: The Schur multiplicative and harmonic convexities of the complete symmetric function. Math. Nachr. 284(5-6), 653-663 (2011)

4. Chu, Y.M., Wang, M.K.: Optimal Lehmer mean bounds for the Toader mean. Results Math. 61(3-4), 223-229 (2012)

5. Chu, Y.M., Wang, M.K., Qiu, S.L.: Optimal combinations bounds of root-square and arithmetic means for Toader mean. Proc. Indian Acad. Sci. Math. Sci. 122(1), 41-51 (2012)

6. Chu, Y.M., Xia, W.F., Zhang, X.H.: The Schur concavity, Schur multiplicative and harmonic convexities of the second dual form of the Hamy symmetric function with applications. J. Multivar. Anal. 105, 412-421 (2012)

7. Deng, Y., Wu, S., He, D.: A sharpened version of Hardy's inequality for parameter $p=5 / 4$. J. Inequal. Appl. 2013, 63 (2013)

8. Du, G.W., Li, F.: Global higher integrability of solutions to subelliptic double obstacle problems. J. Appl. Anal. Comput. $8(3), 1021-1032(2018)$ 
9. Du, G.W., Li, F.: Interior regularity of obstacle problems for nonlinear subelliptic systems with VMO coefficients. J. Inequal. Appl. 2018, 53 (2018)

10. Evans, L.C.: Partial Differential Equations, 2nd edn. Grad. Stud. Math., vol. 19. Am. Math. Soc., Providence (2010)

11. Feng, Y.H., Liu, C.M.: Stability of steady-state solutions to Navier-Stokes-Poisson systems. J. Math. Anal. Appl. 462, 1679-1694 (2018)

12. Hardy, G.H., Littlewood, J.E., Polya, G.: Inequalities, 2nd edn. Cambridge University Press, Cambridge (1952)

13. Ivanov, S.P., Vassilev, D.N.: Extremals for the Sobolev Inequality and the Quaternionic Contact Yamabe Problem. World Scientific, Hackensack (2011)

14. Kannappan, P.: Functional Equations and Inequalities with Applications. Springer, New York (2009)

15. Li, F: Global existence and uniqueness of weak solution for nonlinear viscoelastic full Marguerre-von Karman shallow shell equations. Acta Math. Sin. Engl. Ser. 25(12), 2133-2156 (2009)

16. Li, F.: Limit behavior of the solution to nonlinear viscoelastic Marguerre-von Karman shallow shells system. J. Differ. Equ. 249, 1241-1257 (2010)

17. Li, F., Bai, Y.: Uniform rates of decay for nonlinear viscoelastic Marguerre-von Karman shallow shell system. J. Math Anal. Appl. 351(2), 522-535 (2009)

18. Li, F., Bao, Y.: Uniform stability of the solution for a memory-type elasticity system with nonhomogeneous boundary control condition. J. Dyn. Control Syst. 23, 301-315 (2017)

19. Li, F., Du, G.: General energy decay for a degenerate viscoelastic Petrovsky-type plate equation with boundary feedback. J. Appl. Anal. Comput. 8, 390-401 (2018)

20. Li, F., Gao, Q.: Blow-up of solution for a nonlinear Petrovsky type equation with memory. Appl. Math. Comput. 274 383-392 (2016)

21. Li, F., Hu, F.Y.: Weighted integral inequality and applications in general energy decay estimate for a variable density wave equation with memory. Bound. Value Probl. 2018, 164 (2018)

22. Li, F., Zhao, C.: Uniform energy decay rates for nonlinear viscoelastic wave equation with nonlocal boundary damping. Nonlinear Anal., Theory Methods Appl. 74, 3468-3477 (2011)

23. Li, F., Zhao, Z., Chen, Y.: Global existence uniqueness and decay estimates for nonlinear viscoelastic wave equation with boundary dissipation. Nonlinear Anal., Real World Appl. 12, 1770-1784 (2011)

24. Liu, C.M., Peng, Y.J.: Stability of periodic steady-state solutions to a non-isentropic Euler-Maxwell system. Z. Angew. Math. Phys. 68, 105 (2017)

25. Liu, C.M., Peng, Y.J.: Convergence of a non-isentropic Euler-Poisson system for all time. J. Math. Pures Appl. 119(9), 255-279 (2018)

26. Pachpatte, B.G.: Integral and Finite Difference Inequalities and Applications. Elsevier, Amsterdam (2006)

27. Patriksson, M.: Nonlinear Programming and Variational Inequality Problem. Kluwer Academic Publishers, Dordrecht (1999)

28. Pons, O.: Inequalities in Analysis and Probability. World Scientific, Hackensack (2013)

29. Qian, W.M., Chu, Y.M.: Sharp bounds for a special quasi-arithmetic mean in terms of arithmetic and geometric means with two parameters. J. Inequal. Appl. 2017, 274 (2017)

30. Qin, Y.: Analytic Inequalities and Their Applications in PDEs. Springer, Switzerland (2017)

31. Qin, Y.M.: Analytic Inequalities and Their Applications. Springer, Cham (2017)

32. Steinbach, J: A Variational Inequality Approach to Free Boundary Problem with Applications. Birkhäuser, Basel (2002)

33. Wang, F.Y.: Harnack Inequalities for Stochastic Partial Differential Equations. Springer, New York (2013)

34. Wang, G.D., Zhang, X.H., Chu, Y.M.: A power mean inequality for the Grötzsch ring function. Math. Inequal. Appl. 14(4), 833-837 (2011)

35. Wang, M.K., Chu, Y.M.: Landen inequalities for a class of hypergeometric functions with applications. Math. Inequal. Appl. 21(2), 521-537 (2018)

36. Wang, M.K., Chu, Y.M., Qiu, Y.F., Qiu, S.L.: An optimal power mean inequality for the complete elliptic integrals. Appl. Math. Lett. 24(6), 887-890 (2011)

37. Wang, M.K., Li, Y.M., Chu, Y.M.: Inequalities and infinite product formula for Ramanujan generalized modular equation function. Ramanujan J. 46(1), 189-200 (2018)

38. Wang, M.K., Qiu, S.L., Chu, Y.M.: Infinite series formula for Hübner upper bound function with applications to Hersch-Pfluger distortion function. Math. Inequal. Appl. 21(3), 629-648 (2018)

39. Wang, M.K., Wang, Z.K., Chu, Y.M.: An optimal double inequality between geometric and identric means. Appl. Math. Lett. 25(3), 471-475 (2012)

40. Xu, Q., Zhou, M., Zhang, X.: On a strengthened version of Hardy's inequality. J. Inequal. Appl. 2012, 300 (2012)

41. Yang, Z.H., Qian, W.M., Chu, Y.M., Zhang, W.: On approximating the error function. Math. Inequal. Appl. 21(2), 469-479 (2018)

42. Yang, Z.H., Qian, W.M., Chu, Y.M., Zhang, W.: On approximating the arithmetic-geometric mean and complete elliptic integral of the first kind. J. Math. Anal. Appl. 462(2), 1714-1726 (2018)

43. Yang, Z.H., Zhang, W., Chu, Y.M.: Sharp Gautschi inequality for parameter $0<p<1$ with applications. Math. Inequal. Appl. 20(4), 1107-1120 (2017)

44. Zhang, J.: Extensions of Hardy inequality. J. Inequal. Appl. 2006, Article ID 69379 (2006)

45. Zhao, T.H., Wang, M.K., Zhang, W., Chu, Y.M.: Quadratic transformation inequalities for Gaussian hypergeometric function. J. Inequal. Appl. 2018, 251 (2018) 